\title{
Anatomía Microquirúrgica e Irrigación Arterial de la Región Uncal
}

\author{
Microsurgical Anatomy and Arterial Irrigation of the Uncal Region
}

\begin{abstract}
Rafael Avendaño Pradel"; J. Jesus Martínez Anda**; Mariano Torres Villanueva***; Carlos Castillo Rangel ${ }^{\text {*****; }}$; Carolina Escobar Briones $^{* * * * * *}$; Diego Pineda Martínez ${ }^{* * * * * *}$ \& Sebastián Manuel Arteaga Martínez ${ }^{* * * * * * * *}$
\end{abstract}

AVENDAÑO, P. R.; MARTÍNEZ, A. J. J.; TORRES, V. M.; CASTILLO, R. C.; ESCOBAR, B. C.; PINEDA, M. D. \& ARTEAGA, M. S. M. Anatomía microquirúrgica e irrigación arterial de la región uncal. Int. J. Morphol.,34(2):784-787, 2016.

RESUMEN: El objetivo de este trabajo fue realizar una revisión microquirúrgica de los principales ramos arteriales que otorgan irrigación a la región uncal, identificando sus principales variantes y sus relaciones anatómicas mas relevantes con las estructuras circundantes. Se estudiaron 20 hemisferios cerebrales con el sistema arterial perfundido con latex y colorante mediante disección microquirúrgica y bajo aumento con un rango de $3 \mathrm{X}$ a $40 \mathrm{X}$. Se realizaron registros morfométricos de las principales estructuras. La irrigación de la región uncal del lóbulo temporal se establece principalmente por tres grupos de ramas uncales: las ramas uncales anteriores provenientes de la arteria temporopolar que es uno de las ramas colaterales que inicialmente se derivan de la arteria cerebral media en su segmento M1. Ramas uncales mediales provenientes de la arteria coroidea anterior en su trayecto cisternal. Ramas uncales posteriores provenientes de los segmentos P2A y P2P de la arteria cerebral posterior. La relevancia de la descripción vascular arterial de la región uncal radica en la aplicación del conocimiento de estas relaciones y variantes durante los diversos procedimientos diagnósticos y quirúrgicos del lóbulo temporal.

PALABRAS CLAVE: Rama uncal; Arteria uncal; Arterias del hipocampo; Arteria coroidea anterior.

\section{INTRODUCCIÓN}

El lóbulo temporal comprende cuatro superficies o caras, de las cuales la superficie medial ha sido dividida para su estudio por Fernández-Miranda et al. (2010), en un segmento anterior, uno medio y uno posterior. El segmento anterior comprende al uncus y la corteza entorrinal y se extiende desde donde el surco rinal se torna superiormente en el límite anterior del uncus hasta su límite posterior establecido por el surco colateral. El límite posterior del área entorrinal se acepta como el límite posterior del uncus. El uncus comprende un segmento anterior y uno posterior divididos por una prominencia de dirección medial, el ápex del uncus. El segmento anterior comprende a los giros semilunar y ambiente, mientras el segmento posterior dividido en porciones superior e inferior por el surco uncal, la porción inferior esta formada por el giro del parahipocampo mientras que la superior comprende tres pequeños giros: el giro uncinado, la banda de Giacomini como continuación del giro dentado y el giro intralímbico.

La irrigación arterial de la región uncal del lóbulo temporal, comprende un territorio en donde diversos ramos arteriales participan, estos ramos se ha identificado provienen de las principales afluentes arteriales que circundan la región y suelen originarse de la arteria coroidea anterior, de

Servicio de Neurocirugía, Hospital Regional $1^{\circ}$ de Octubre, Instituto de Seguridad y Servicios Sociales de los Trabajadores del Estado (ISSSTE). Departamento de Anatomía Humana. Facultad de Medicina, Universidad Nacional Autónoma de México (UNAM), México D.F., México.

** Centro Neurológico. Hospital ABC, Departamento de Anatomía, Facultad de Medicina. Universidad Nacional Autónoma de México (UNAM), México D.F., México.

**** Servicio de Neurocirugía. Hospital de Especialidades. "Dr. Antonio Fraga Mouret.” Centro Médico Nacional. La Raza. Instituto Mexicano del Seguro Social (IMSS), México D.F., México.

***** Profesor Titular del Curso de Neurocirugía. Hospital Regional $1^{\circ}$ de Octubre, Instituto de Seguridad y Servicios Sociales de los Trabajadores del Estado (ISSSTE), México D.F., México.

****** Coordinadora de Investigación. Laboratorio de Ritmos Biológicos y Metabolismo. Departamento de Anatomía. Facultad de Medicina, Universidad Nacional Autónoma de México (UNAM), México D.F., México.

******** Jefe del Departamento de Anfiteatro. Facultad de Medicina, Universidad Nacional Autónoma de México (UNAM), México D.F., México.

********Jefe del Departamento de Anatomía. Facultad de Medicina, Universidad Nacional Autónoma de México (UNAM), México D.F., México. 
la porción supraclinoidea de la arteria carótida interna en su segmentos coroideo y comunicante, de los ramos tempranos de la arteria cerebral media y ramas temporopolares, así como de los primeras ramas corticales de la arteria cerebral posterior (Wen et al., 1999). En el estudio clásico de Erdem et al. (1993), la vasculatura hipocampal se divide en 5 grupos en orden de frecuencia: Grupo A con $57 \%$ de frecuencia con un origen mixto de la Arteria coroidea anterior (AchoA), de la arteria cerebral posterior (ACP), temporal inferior, coroidea posterior y de los ramos espleniales de la ACP. Grupo B con $27 \%$ con todos los ramos temporales inferiores de ACP irrigando predominantemente al hipocampo. Grupo $\mathrm{C}$ en $10 \%$ la rama temporal anteroinferior de la $\mathrm{ACP}$ como afluente arterial dominante. Grupo D en $3 \%$ el hipocampo es irrigado por arterias que se originan directo de la ACP (Arteria de Uchimura). Grupo E en $3 \%$ la arteria hipocampal se origina directo de la AChoA. Huther et al. (1998) definieron 6 grupos arteriales en la región mesial temporal: Grupo I arterias parahipocampales anterosuperiores. Grupo II arterias parahipocampales anteroinferiores. Grupo III arterias uncales mediales. Grupo IV arterias uncales laterales. Grupo V arterias parahipocampales posteriores. Grupo VI arterias hipocampales posteriores. A pesar de que existen diversos estudios sobre la vasculatura de la región uncal, estos presentan diversas variables en los reportes realizados.

El objetivo de esta investigación fue realizar un análisis morfológico post mortem de la vasculatura arterial de la región uncal desde el punto de vista quirúrgico, con la finalidad de aportar información útil para el diseño de mejores técnicas quirúrgicas para el acceso a dicha región cerebral.

\section{MATERIAL Y MÉTODO}

Se utilizaron 10 cerebros humanos extraídos de la bóveda craneana de forma tradicional, dentro del departamento de Anatomía de la Facultad de Medicina de la UNAM y fijados con técnica tradicional de formol al $10 \%$, de los cuales se excluyeron 3 por presentar daño considerable de los sistemas vasculares vertebrobasilar y carotideo. Se empleó hule latex liquido como polímero para perfusión vascular teñido con pintura acrílica roja. Se emplearon para disección microvascular disectores de Rhoton, equipo de microcirugía, equipo de iluminación tipo halógeno, un Microscopio estereoscópico (Nikon, modelo C-FBMN) con el software Infinity Capture (Versión 6.4.0) para documentar imágenes. Una vez completo el proceso de fijación del encéfalo, se procedió a la disección e identificación de ambas arterias carótidas internas y del sistema vertebrobasilar. Se realizó un lavado del sistema vascular canulando e infiltrando ambas carótidas y arterias vertebrales con agua hasta su total limpieza, identificando rupturas vasculares las cuales se ocluyeron para evitar la fuga del polímero. Se perfundió latex líquido teñido con pintura acrílica roja cargado en jeringas de $1 \mathrm{~mL}$ alternando los sistemas carotídeo izquierdo, derecho, así como el sistema vertebrobasilar, hasta obtener la tinción de los principales troncos vasculares cerebrales y sus principales ramos. Se dejaron reposar $24 \mathrm{~h}$ antes de su extracción para disección microscópica.

\section{RESULTADOS}

Del total de la muestra $(n=10)$ se estudiaron siete encéfalos perfundidos (14 hemisferios cerebrales). Se excluyeron tres encéfalos debido a que el sistema arterial cerebral estaba seriamente dañado. La irrigación uncal se puede dividir en tres grupos de ramos principales que se distribuyen sobre la superficie uncal.

Ramas uncales mediales (Fig. 1): La media fue de 3,33 ramas con un rango de 2 a 5 ramas que nacen a través de un tronco principal presente en 13 hemisferios $(92,8 \%)$ que emerge como primera rama colateral de la arteria coroidea anterior, en promedio a $5,4 \mathrm{~mm}$ de su origen con un rango de 2 a $18 \mathrm{~mm}$ para después presentar una división temprana de origen perpendicular al tronco uncal principal. El tronco principal se continúa con un trayecto promedio de $6,5 \mathrm{~mm}$ hasta llegar a la superficie pial de la región anterosuperior del uncus para irrigar en orden de frecuencia a las siguientes estructuras: Giro semilunar, fisura coroidea y ápex uncal, limen de la ínsula y surco rinal.

Ramas uncales anteriores (Fig. 2): La media de estos ramas fue de 2 y provenien de la arteria temporopolar que se aprecia como primera rama temprana de la ACM en 12 hemisferios $(85,7 \%)$ y que nace directo de la bifurcación de la arteria carótida interna (ACI) o del segmento coroideo en dos hemisferios ( $7,14 \%$ respectivamente) y que mide 11,7 $\mathrm{mm}$ en promedio con un rango de 5 a $23 \mathrm{~mm}$, esta discurre a través de la porción esfenoidal del surco lateral del cerebro cerca de la superficie cortical del planum polare se bifurca en dos ramos principales uno lateral y otro medial, este último posee un trayecto muy tortuoso antes de contactar la superficie pial y distribuirse por ramos diminutos en la cara anteroinferior de la región uncal otorgando irrigación a las siguientes estructuras en orden decreciente de frecuencia: polo temporal, limen de la ínsula, surco rinal, corteza entorrinal, surco colateral, giro semilunar y giro uncal.

Ramas uncales posteriores (Fig. 3): Existen 2 ramas en promedio con un rango de 1 a 4 ramas y provienen de las primeras ramas corticales de los segmentos $\mathrm{P} 2 \mathrm{~A}$ y $\mathrm{P} 2 \mathrm{P}$ de la 


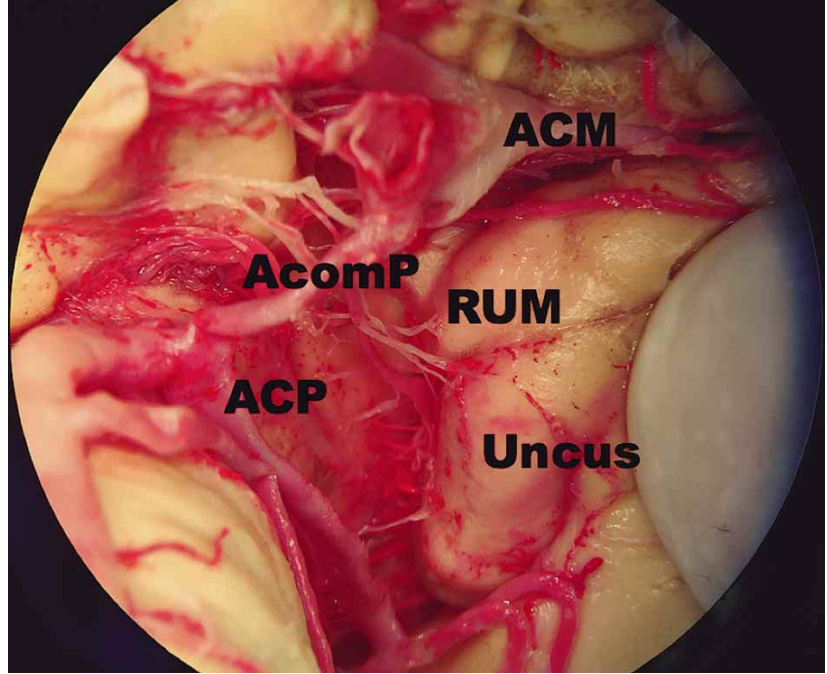

Fig. 1. Visión bajo microscopía de los ramos uncales mediales. $\mathrm{AcomP}=$ arteria comunicante posterior, $\mathrm{ACM}=$ arteria cerebral media, $\mathrm{ACP}=$ arteria cerebral posterior y $\mathrm{RUM}=$ ramas uncales mediales.

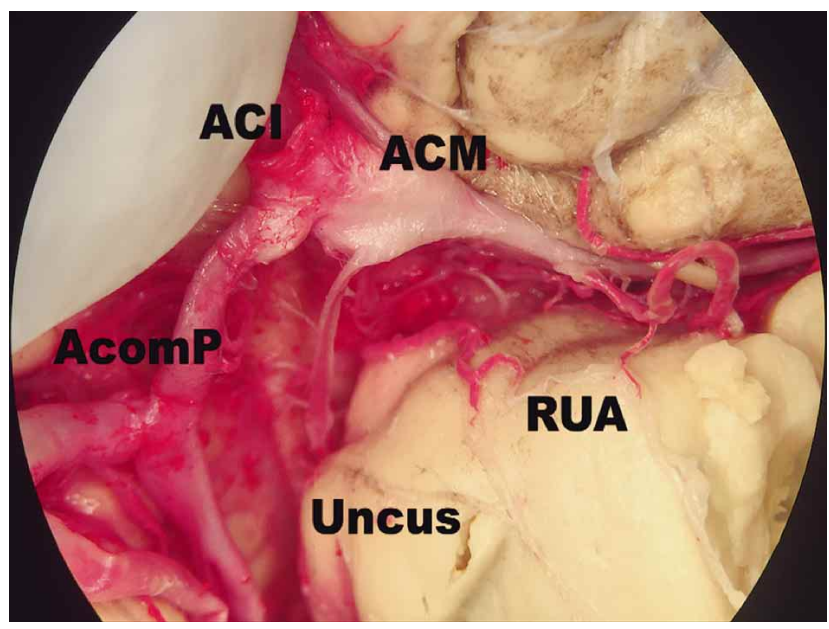

Fig. 2. Visión bajo microscopía de los ramos uncales anteriores. $\mathrm{ACI}=$ arteria carótida interna, $\mathrm{ACM}=$ arteria cerebral media, $\mathrm{AcomP}=$ arteria comunicante posterior y $\mathrm{RUA}=$ ramas uncales anteriores.

ACP, y nace en promedio a 16,3 mm con un rango de 6 a 22 $\mathrm{mm}$, de la desembocadura de la arteria comunicante posterior $(\mathrm{AcomP})$ a través de un pequeño tronco de aproximadamente $3 \mathrm{~mm}$ promedio con un rango de $1 \mathrm{a} 5 \mathrm{~mm}$ (tronco arterial temporal inferior común), dicho tronco da origen inmediatamente después de su nacimiento a un ramo de 7,4 $\mathrm{mm}$ en promedio con un rango de 4 a $13 \mathrm{~mm}$ de dirección anterosuperior a través de la pared lateral de la cisterna crural que contacta la superficie pial para perderse en la profundidad del surco uncal, esta rama se denomina arteria hipocampal. El tronco temporal inferior común se bifurca

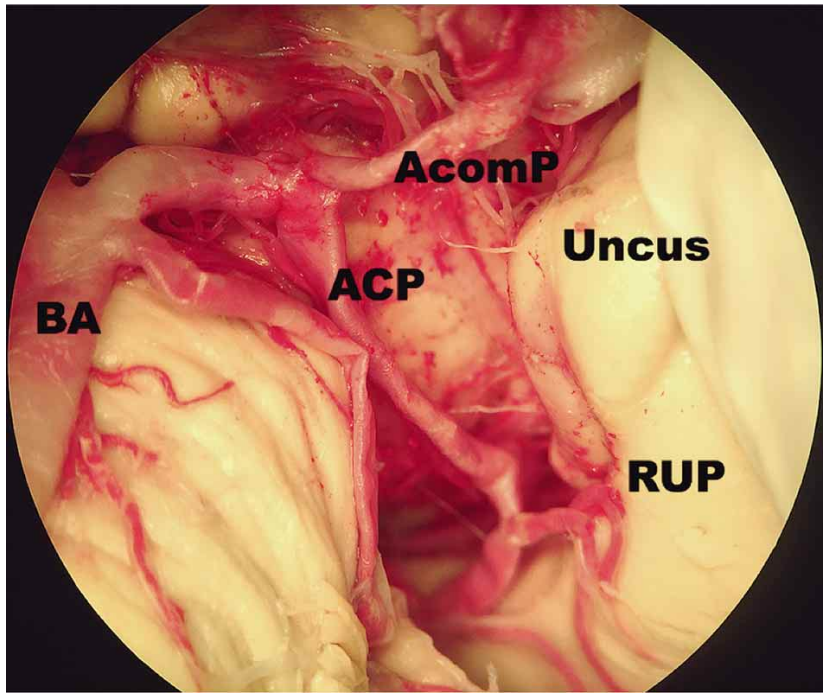

Fig. 3. Visión bajo microscopía de los ramos uncales posteriores. AcomP $=$ arteria comunicante posterior, $\mathrm{ACP}=$ arteria cerebral posterior, $\mathrm{BA}=$ arteria basilar y $\mathrm{RUP}=$ ramas uncales posteriores.

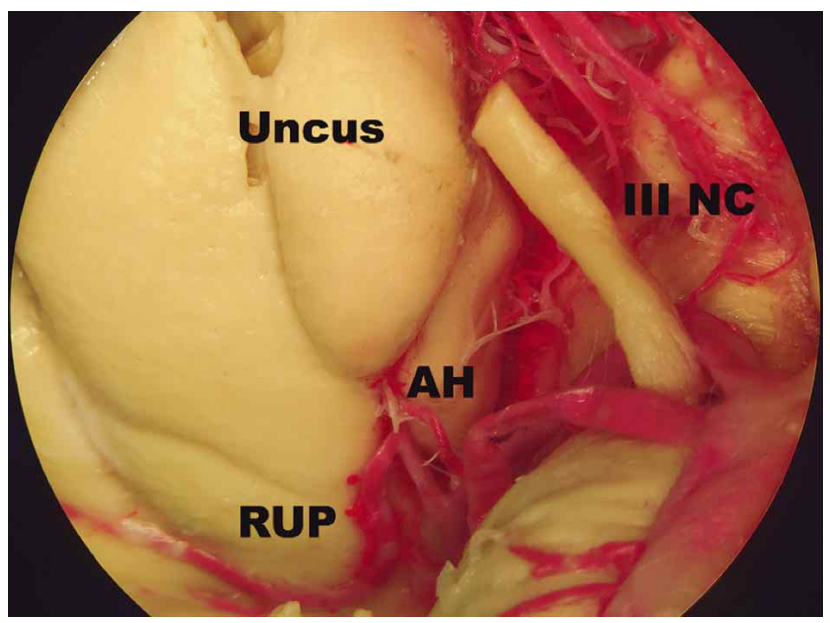

Fig. 4. Visión bajo microscopía de la arteria hipocampal. AH= arteria hipocampal y $\mathrm{RUP}=$ ramas uncales posteriores.

al contactar la superficie pial sobre el giro del parahipocampo dando origen a dos ramos lateral y medial, este último discurre superficialmente hasta distribuirse mediante diminutas ramas en la cara posteroinferior del uncus y que inclusive alcanzan una distribución limítrofe adyacente al surco rinal con el territorio irrigado por la rama medial perteneciente a las ramas uncales anteriores provenientes de la arteria temporopolar. En orden decreciente, las estructuras irrigadas por las ramas uncales posteriores son: el giro uncal, surco uncal, la corteza entorrinal, giro del parahipocampo, giro colateral y surco rinal. 


\section{DISCUSIÓN}

El presente estudio post mortem aborda la anatomía vascular arterial de la región uncal, de forma diferente a las descripciones clásicas, y acorde a un modelo prismático de la región uncal, presenta principalmente tres superficies, una anterior y dos posteriores sobre las cuales se distribuyen estos tres principales grupos de ramas uncales. Cabe resaltar la gran variabilidad que existe entre los grupos de ramas uncales anteriores y mediales, no solo en el número de ramas o en el territorio irrigado, si no en el sitio de donde se originan, ya que en algunos hemisferios se apreciaron ramas directas desde la bifurcación o a partir del segmento coroideo de la ACI, en contraste con la distribución constante y uniforme que presenta el sistema de ramos uncales posteriores en los que la variabilidad fue mucho menor.

La arteria hipocampal (Fig. 4) prevalece como uno de los ramos posteriores que generalmente se origina a partir de un tronco temporal inferior, sin embargo en dos hemisferios se logró apreciar como rama directa de la arteria coroidea anterior. Las limitaciones de este estudio son principalmente relacionadas al manejo del material biológico y calidad del mismo acorde a la técnica de extracción y conservación, así como de los factores relacionados a la técnica de perfusión vascular sin embargo continúa siendo la forma más fidedigna de estudiar el sistema vascular cerebral de interés constante para el neurocirujano.

AVENDAÑO, P. R.; MARTÍNEZ, A. J. J.; TORRES, V. M.; CASTILLO, R. C.; ESCOBAR, B. C.; PINEDA, M.D. \& ARTEAGA, M. S. M. Microsurgical anatomy and arterial irrigation of the uncal region. Int. J. Morphol., 34(2):784-787, 2016.

SUMMARY: The objective of this work was realizing a microsurgical review of the main arterial ramus that distribute irrigation to the uncal region, identifying the most common variations and more relevant relationships with surrounding structures. Twenty (20) fixed human brain hemispheres were studied, with the arterial latex and red colorant perfusion technique for dissection under microscope magnification (3X-40X). Morphometric characterization and data were obtained of the structures studied. Arterial irrigation of the uncal region of the temporal lobe is established by three groups of uncal ramus: the anterior uncal rami, deriving from the temporopolar artery, which is one of the first branches of the middle cerebral artery in segment M1. The medial uncal rami, branches of the cisternal portion of the anterior choroidal artery. The posterior uncal rami, branches of the $\mathrm{P} 2 \mathrm{~A}$ and $\mathrm{P} 2 \mathrm{P}$ segments of the posterior cerebral artery. The relevance of arterial vascular description of the uncus, results in the application of knowledge of the variations and relationships during the diagnostic and surgical procedures of the temporal lobe.

KEY WORDS: Uncal ramus; Uncal artery; Hippocampal artery; Anterior choroidal artery.

\section{REFERENCIAS BIBLIOGRÁFICAS}

Erdem, A.; Yasargil, G. \& Roth, P. Microsurgical anatomy of the hippocampal arteries. J. Neurosurg., 79(2):256-65, 1993.

Fernández-Miranda, J. C.; de Oliveira, E.; Rubino, P. A.; Wen, H. T. \& Rhoton, A. L. Jr. Microvascular anatomy of the medial temporal region: part 1: its application to arteriovenous malformation surgery. Neurosurgery, 67(3 Suppl Operative):ons237-76, 2010.

Huther, G.; Dörlf, J.; Van der Loos, H. \& Jeanmonod, D. Microanatomic and vascular aspects of the temporomesial region. Neurosurgery, 43(5):1118-36, 1998.

Wen, H. T.; Rhoton, A. L. Jr.; de Oliveira, E.; Cardoso, A. C.; Tedeschi, H.; Baccanelli, M. \& Marino, R. Jr. Microsurgical anatomy of the temporal lobe: part 1: mesial temporal lobe anatomy and its vascular relationships as applied to amygdalohippocampectomy. Neurosurgery, 45(3):549-91, 1999.

Dirección para Correspondencia:

Dr. Rafael Avendaño Pradel

Servicio de Neurocirugía

Hospital Regional 10 de Octubre

Instituto de Seguridad y Servicios Sociales de los Trabaja-

dores del Estado (ISSSTE)

Departamento de Anatomía, Facultad de Medicina

Universidad Nacional Autónoma de México (UNAM)

México D.F.

MÉXICO

Email: rafapradel@icloud.com

Recibido : 07-12-2015

Aceptado: 06-04-2016 